\title{
Cambios paradigmáticos en la formación de profesores: Peligros, trampas y la promesa no cumplida del profesional reflexivo
}

\author{
Paradigmatic Changes in Teacher Education: \\ The Perils, Pitfalls, and Unrealized Promise of the \\ Reflective Practitioner
}

\section{Les changements paradigmatiques en éducation des enseignants : Les périls, les pièges et la promesse non réalisée d'un praticien réflexif}

\author{
Tom Russell \\ Queen's University, Canadá
}

\begin{abstract}
RESUMEN
Los programas de formación inicial docente tienden a estar saturados con referencias a la reflexión, a la práctica reflexiva y a la importancia de volverse un profesional crítico y reflexivo. A más de 25 años desde que los educadores comenzaron a considerar el concepto de Schön, hay poca evidencia de que el potencial de su idea de un profesional reflexivo se haya desarrollado en nuestras escuelas y programas de formación pedagógica. Este capitulo hace una revisión de literatura especializada sobre la práctica reflexiva en la formación inicial docente para construir una explicación acerca del hecho que los tan anhelados cambios paradigmáticos no han ocurrido. La complejidad de cambios paradigmáticos, la estabilidad de las prácticas docentes, y la significatividad del aprendizaje por observación que todos los profesores y formadores de profesores traen consigo a su trabajo son considerados en la construcción de una explicación de los muchos peligros y trampas asociadas al incumplimiento de la promesa del concepto de profesional reflexivo en la formación de profesores.
\end{abstract}

Descriptores: cambios paradigmáticos en los programas de formación de maestros y profesores de media; profesional reflexivo crítico. 


\begin{abstract}
Preservice teacher education programs tend to be saturated with references to reflection, reflective practice, and the importance of becoming a critically reflective practitioner. More than 25 years since teacher educators began to take up Schön's term, there is little evidence that the potential of his idea of a reflective practitioner has been realized in our schools and teacher education programs. This chapter reviews selected literature on reflective practice in teacher education to build an explanation for the fact that the desired paradigmatic changes have largely failed to happen. The complexity of paradigm changes, the stability of teaching practices, and the significance of the apprenticeship of observation that all teachers and teacher educators bring to their work are considered in constructing an explanation for the many perils and pitfalls associated with failing to achieve the promise of the reflective practitioner concept in teacher education.
\end{abstract}

Key words: paradigmatic changes in teacher education; critically reflective practitioner.

\title{
RÉSUMÉ
}

Les programmes d'éducation pour enseignants en pré service ont tendance à être saturés de références à la réflexion, à la pratique de la réflexion et à l'importance de devenir des praticiens réflexifs critiques. Plus de vingt-cinq ans après que les éducateurs des enseignants aient entrepris d'adopter ce terme de Schön, il y a peu d'évidence que le potentiel de son idée de praticien réflexif se soit réalisé dans nos écoles et dans les programmes d'éducation des enseignants. Ce chapitre examine la littérature choisie sur la pratique réflexive en éducation des enseignants, afin d'expliquer pourquoi les changements paradigmatiques désirés n'ont pas réussi à se concrétiser. Les facteurs de complexité des changements paradigmatiques, de stabilité des pratiques d'enseignement et de l'importance de l'apprentissage à l'observation, que tous les enseignants et éducateurs d'enseignants apportent à leur tâche, sont pris en considération pour élaborer une explication au sujet des multiples périls et pièges associés à la non réussite du concept de praticien réflexif en éducation des enseignants en vue d'expliquer pourquoi la promesse ne s'est pas réalisée.

Mots clés : Changements paradigmatiques dans l'éducation des enseignants; les praticiens réflexifs critiques.

$\mathrm{E}^{\mathrm{s}}$ PROPÓSITO PRINCIPAL en este capítulo es problematizar el uso virtualmente universal de frases como reflexión, práctica reflexiva y convertirse en un profesional reflexivo en la formación profesional en general, y en la formación inicial docente en particular. La introducción de estos términos es amplia y apropiadamente atribuida a Donald Schön que publicó tres libros acerca del tema. El primer libro fue El profesional reflexivo: como piensan los profesionales cuando actúan, publicado en $1983 .{ }^{1}$ En 1987 fue seguido por el segundo, Educando al profesional reflexivo: hacia un nuevo diseño para la enseñanza y el aprendizaje en las profesiones. Luego en 1990, publica el tercero que es una colección editada bajo el título El giro reflexivo: estudios de caso en y sobre prácticas educativas. Una de las últimas contribuciones de Schön fue un artículo del año 1995 intitulado El nuevo saber requiere de una nueva epistemología.

Hay muchos aspectos en los tres libros de Schön que los formadores de profesores parecen haber ignorado o interpretado de manera imprecisa. Schön no estaba escribiendo desde una perspectiva de formación docente, sino que sugería que si uno estuviese buscando ejemplos de reflexión en la acción, uno de los últimos lugares donde uno podría encontrarlos sería en una escuela. Quizás el subtítulo de su primer libro 
se acerca más directamente a indicar su enfoque: cómo piensan los profesionales cuando actúan. Ese primer libro incluye una serie de capítulos ilustrativos desde profesiones tan diversas como la arquitectura, la psicoterapia y la planificación urbanística; ninguno de los capítulos ilustra el trabajo de un profesor, aunque en algunas conferencias públicas Schön sí hace referencias al trabajo de algunos profesores con los que él estaba familiarizado.

$\mathrm{El}$ argumento en este capítulo puede resumirse de la siguiente manera: Reflexión es una palabra cotidiana que puede ser interpretada de muchas formas. Si hacemos caso omiso de sus interpretaciones físicas, la mayoría de los significados de reflexión que quedan se refieren a actividades mentales que pueden parecer simples y directas porque frecuentemente se asocian a actividades relajadas o que no requieren mayor esfuerzo. Mientras que Schön $(1983,1987)$ se centró en lo que llamó reflexión en la acción y sus implicancias para el aprendizaje profesional, los argumentos y sus aplicaciones en el contexto de formación de profesores parece prestarle poca o ninguna atención al enfoque de Schön en el replanteamiento y las implicaciones correspondientes al cambio en la actuación profesional.

En este capítulo, se revisan los argumentos de una serie de publicaciones sobre el tema de la reflexión en la formación de profesores para ilustrar esta conclusión. Luego, se desarrolla una interpretación personal de las implicaciones del trabajo de Schön para la formación docente y las oportunidades para mejorar la formación del profesorado que parecen estar perdiéndose al ignorar esas implicaciones. A esta altura de la discusión resulta útil presentar los tres puntos que -a mi parecer-son fundamentales en los argumentos de Schön y que aparecen en el libro que dio inicio al enamoramiento de la formación docente con la reflexión (crítica). Los siguientes puntos son una interpretación personal que quizás simplifica demasiado los argumentos:

1. La tendencia a llevar la teoría a la práctica, tan profundamente arraigada en la cultura occidental (resumida por la frase racionalidad técnica), resulta engañosa e inapropiada cuando se aplica a situaciones de acción profesional y aprendizaje desde la experiencia.

2. Normalmente nos concentramos en solucionar un problema sin antes considerar si hemos primero, identificado apropiadamente ese problema. Los problemas no aparecen con una etiqueta que los presente como tales. La manera en que nombramos un problema influye significativamente en la manera en que intentamos resolverlo.

3. Formas alternativas de plantear un problema pueden surgir espontáneamente en cualquier contexto de acción profesional, particularmente en momentos de incertidumbre, perplejidad o sorpresa. Escuchar y dialogar con la situación y los individuos involucrados en ella puede estimular nuevas formas de interpretar los problemas que sugieren diversas alternativas de acción. Éstas podrían resultar más o menos productivas, y por lo tanto (necesitan ser testeadas) habrá que examinarlas en la acción. 


\section{Contexto Personal}

Comencé a enseñar como profesor voluntario y sin entrenamiento en el año 1963 en Nigeria. En esos dos primeros años de enseñanza, estuve trabajando desde mi aprendizaje basado en la observación (Lortie, 1975), y actuaba) de la manera en la que había visto actuar a mis propios profesores. No se me había enseñado a cómo enseñar formalmente, de manera contextualizada por la escuela, mas yo sí sabía después de una prolongada observación) de qué manera se comportaban los profesores y qué estrategias básicas empleaban en el salón de clases. Ciertamente no era un gran profesor -tampoco era un desastre- pero ese largo período de desinformada enseñanza no logró formar hábitos que luego no pudiera cambiar. Esas experiencias como profesor no formado me dejaron ansioso por conocer más el contenido de un programa de formación inicial docente; el proceso de enseñanza que vino más tarde fue no sólo exitoso, sino también memorable. Luego de terminar mis estudios de Doctor en Filosofía, $\mathrm{PhD}$, comencé a enseñar a estudiantes de educación de profesor de preservicio en el año 1977. Los tres años precedentes a esa experiencia, trabajé con profesores con mucha experiencia en una variedad de contextos de formación continua del profesorado. Este trabajo con profesores experimentados moldeó mis expectativas con respecto a los profesores en formación, y el modo en que debería enseñarles. Mi interés estaba más enfocado en ayudar a estos estudiantes a aprender de sus propias experiencias en las prácticas profesionales, en lugar de llenarlos con ideas potencialmente irreales acerca de cómo deberían trabajar con sus estudiantes.

En mi sexto año trabajando como formador de profesores, llegó a mis manos el libro "El profesional reflexivo", justo luego de su publicación en 1983. El tratamiento que Schön hace acerca de la reflexión en la acción, tocó profundamente en un área que era sensible para mí, al ver que cada vez que visitaba una escuela para hacer observaciones de práctica, veía muy poca evidencia de las prácticas que tanto yo como otros profesores estábamos enseñando en la universidad. Parecía que lo que los estudiantes estaban haciendo era no aplicar la teoría en la práctica. El año sabático que tomé me dio la oportunidad de explorar la idea de reflexión en la acción en el contexto de un programa de formación que estuve visitando. De manera fortuita, un colega en el año de 1984, invitó a Donald Schön a dar tres conferencias en la Universidad Queen's, justo un poco después de mi regreso a la facultad. Esas conferencias fueron las más memorables a las que he asistido $y$, me inspiraron a tomar la perspectiva de Schön más seriamente y a conferirle más importancia de la que otros formadores de profesores parecían darle.

Ya en 1985 era evidente que muchos otros formadores de docentes se estaban interesando en la reflexión como un término que podría ser aplicado en el contexto de formación docente. Sorpresivamente, las bitácoras que los profesores les habían estado pidiendo a sus estudiantes comenzaron a ser remplazadas por tareas que requerían explícitamente de reflexión. Mientras me dedicaba a trabajar para explorar el significado de la reflexión en la acción por medio de una serie de investigaciones, gradualmente me empecé a dar cuenta que mi interpretación personal del término difería significativamente de las interpretaciones que estaban apareciendo en la literatura. 
En este capítulo exploro una pequeña selección del vasto número de artículos que se han publicado sobre el tema, enfocándome en la manera en la que los términos reflexión y reflexión en la acción han sido interpretados en formas diversas y por propósitos diferentes. Luego discuto la interpretación que continúo encontrando más productiva en mi propia práctica como formador de profesores.

\section{Seis Perspectivas sobre la reflexión y la formación docente}

\section{Linda Valli: Casos y críticas de la formación reflexiva de profesores}

La colección de siete estudios de caso de Valli (1992) sobre programas de formación docente enfocados en varias formas de reflexión está acompañada de seis críticas desde una diversidad de perspectivas. Este libro provee uno de nuestros mejores recursos para comprender la complejidad de lo que está involucrado en el desarrollo de la reflexión de manera significativa en un programa de formación inicial docente. Aunque los estudios de caso describen programas tal como eran hace dos décadas atrás, aún así ilustran claramente algunas de las muchas maneras en que la reflexión puede ser interpretada al interior de programas de formación docente. Los casos también describen varias estructuras de práctica profesional desde la perspectiva de fomentar la reflexión.

Desafortunadamente, y tal como en la mayoría de los artículos publicados sobre la reflexión y la práctica reflexiva en la educación de docentes, hay muy pocas referencias directas y específicas a la práctica en pregrado en las seis críticas que ofrecen varios modos de interpretación de los siete estudios de caso. La crítica de Calderhead (1992) extrajo ricas conclusiones, pero hay poca evidencia en la literatura como para indicar que sus consejos hayan sido practicados:

"Hay pocas directrices bien establecidas para los formadores de profesores en esta nueva área, y como resultado se les pide constantemente a los tutores y profesores supervisores que examinen lo que están haciendo y hacia donde están yendo. Los formadores de profesores se han impuesto ambiciosos objetivos en la formación reflexiva, pero aún es incierto qué es lo realmente alcanzable en el contexto de un programa de formación inicial docente" (p. 145).

"[Las preguntas acerca de qué es lo posible] solo pueden ser respondidas por medio de la experiencia de los formadores, y la evaluación de esa experiencia, en el desarrollo de programas de formación inicial para la práctica reflexiva y en la promoción de nuestra(s) comprensión(es) del crecimiento profesional. En el proceso de desarrollar una enseñanza reflexiva como meta de la formación inicial, existe la necesidad de desarrollar nuestro propio conocimiento acerca de la enseñanza reflexiva y cómo puede ser facilitada" (pp. 145-146). 
"Antes que podamos tener profesores reflexivos, necesitamos escuelas reflexivas y formadores de docentes reflexivos" (p. 146).

Las palabras de Calderhead ayudan a resumir la contribución de los casos y críticas como las que aparecen en el texto de Valli. Los formadores de docentes necesitan experimentar cambios paradigmáticos en ellos mismos antes de esperar que los estudiantes de pregrado hagan lo mismo. Los profesores de los programas de formación docente necesitan explorar de qué manera las discusiones sobre práctica reflexiva - como las de Dewey y Schön — se relacionan con sus propias prácticas tanto personales como profesionales en los programas de pedagogía de nuestras universidades. Podemos hacer mucho más daño que bien al añadir el vocabulario de la reflexión a nuestras enunciaciones de misión y a nuestras tareas, si no van acompañados de un amplio soporte que incluya el modelaje y los vínculos explícitos a las experiencias reales de la práctica de los candidatos a profesores.

\section{Lynn Fendler: ¿Necesitan los profesores reflexivos la ayuda de expertos?}

Debido a su masiva distribución en la revista Educational Researcher, el artículo de Fendler (2005), escrito desde una perspectiva histórica, es un trabajo de lectura esencial en el tema de la reflexión. Fendler inicia su articulo con una simple cita de Zeichner (1996:207):

"No existe el profesor irreflexivo." Esta frase, a menudo se cita de forma errónea cuando es tomada fuera de contexto y cuando se omiten las oraciones anteriores y posteriores. Una cita completa dice así: "Primero, uno debe reconocer que en cierto sentido, todos los profesores son reflexivos. No existe el profesor irreflexivo. Necesitamos movernos más allá de la celebración acrítica de la reflexión docente y del empoderamiento docente y centrar nuestra atención en qué tipo de reflexión están involucrándose los profesores, que aquello sobre lo que están reflexionando y cómo les está yendo en eso” (p. 207).

La cita de Fendler parece sugerir que todos los profesores son reflexivos. La cita más completa, con las críticas palabras "en cierto sentido", apunta a la importancia del contexto y a los procesos de las reflexiones de los profesores.

Como muchos que quieren interpretar a Schön en el tema de la reflexión, Fendler vuelve a Dewey, tal como el mismo Schön lo hizo. Rápidamente, Fendler establece la conexión entre reflexión docente y el mejoramiento de la enseñanza, lo que muchos suponen es el objetivo de la reflexión. De esta manera vemos que la reflexión no es un fin en sí mismo, sino un medio para el fin del mejoramiento, y se asume que la mayoría de los profesionales están interesados en mejorar sus prácticas personales cada vez que les sea posible. Fendler sugiere que su articulo ofrece dos contribuciones a la literatura - una perspectiva histórica y una perspectiva basada en la sociología y en el trabajo de Foucault. 
En la conclusión del artículo, mientras sigue sugiriendo que todos los profesores son reflexivos, Fendler plantea una pregunta crucial:

\begin{abstract}
"Si los investigadores de la educación creen que todos los profesores piensan sobre lo que hacen, entonces por qué se habla tanto acerca de formar profesionales reflexivos?" (p. 23). Fendler continúa: "resulta irónico que la retórica acerca de los profesionales reflexivos se enfoque en empoderar a los profesores, y que los requisitos para aprender a ser reflexivo se encuentren sobre la base del supuesto de que los profesores sean incapaces de reflexionar sin la dirección de autoridades expertas. El caso de la reflexión docente provee un ejemplo de la necesidad que tienen los investigadores de revisar sus propios supuestos acerca de la relación entre la investigación y la formación de profesores (p. 23).
\end{abstract}

Sí bien estoy de acuerdo en que existen muchos supuestos que deben ser examinados, particularmente en lo referente a los profesores como profesionales reflexivos y el significado de la práctica reflexiva para el desarrollo docente, mi enfoque no está en el empoderamiento de los profesores ni en que si la práctica reflexiva requiere de la guía de expertos. El análisis de las tareas de la enseñanza desde la perspectiva de la reflexión en la acción de Schön, y el desarrollo de nuevas prácticas basadas en una reformulación estimulada por la interpretación de las acciones de los estudiantes no es algo que ocurra en la mayoría de los profesores.

\title{
Ken Zeichner: La práctica reflexiva para el avance de la democracia.
}

Zeichner es bien conocido por la mayoría de los formadores de profesores, su extensa bibliografía incluye muchas discusiones sobre la práctica reflexiva en el contexto de la formación docente. En un capítulo de un libro publicado en 1996 titulado Profesores como profesionales reflexivos y la democratización de la reforma escolar, Zeichner ocupa los términos práctica reflexiva y enseñanza reflexiva de (manera indistinta). Èl ve la práctica reflexiva como un "movimiento" (p.200) y utiliza frases como "reflexión sobre la experiencia personal." Luego, a partir de la página 202, Zeichner pasa a discutir "Las cuatro maneras en que se ha empleado el término reflexión en programas de formación docente y que han socavado la intención emancipadora expresada por los formadores de profesores" (p. 202). Las cuatro interpretaciones merecen ser examinadas en mayor detalle:

1. Replicar practicas de investigación.

2. Evitar implicaciones éticas y morales.

3. Evitar consideraciones sociales.

4. Aislar profesores individuales.

Zeichner ve la visualización de la reflexión como la duplicación de las prácticas investigativas (pp. 202-203) como una estrategia para incentivar a los profesores a 
reproducir las prácticas que la investigación empírica llevada a cabo por investigadores universitarios ha demostrado ser efectiva. Atribuye este hecho - correctamente- a la persistencia de una racionalidad técnica, que asume la relación de "teoría sobre la práctica.” Bajo el título, “evitar implicancias éticas y morales” (p. 203-204), Zeichner ve una interpretación de la reflexión que se enfoca en los medios de la enseñanza, pero que descuida los fines de la enseñanza las implicaciones éticas y morales. La siguiente interpretación de reflexión es criticada desde la perspectiva que limita la reflexión, en particular, a la sala de clases, de manera que ignora las consideraciones sociales que afectan el trabajo de los profesores. Finalmente, Zeichner critica las visiones que limitan la reflexión a un acto individual sin la interacción con otros profesores. "Todas estas prácticas ayudan a crear) una situación en la que simplemente existe una mera ilusión de desarrollo y de autonomía docente" (p. 206).

Habiendo alcanzando esta conclusión, Zeichner revela entonces el propósito principal de su capítulo: "La formación de profesores reflexivos que fomenta un desarrollo docente genuino, debe ser apoyada -desde mi perspectiva- sólo si está conectada a la lucha por una mayor justicia social” (p. 206). En pocas palabras, Zeichner parece usar la idea de profesores como profesionales reflexivos como la base para el argumento que la práctica reflexiva sólo debiera ser intencionada en el contexto de reducir las injusticias sociales que son tan visibles en la mayor parte de las sociedades. Su objetivo no es presentar una concepción clara de práctica reflexiva en el contexto de la enseñanza y el aprendizaje, y sin embargo, parece ser leído de esta manera debido a su alto perfil al interior de la comunidad de formación docente.

\section{Neville Hatton y David Smith: Niveles de reflexión que llevan a la reflexión en la acción.}

Hatton y Smith (1995) proveen un análisis detallado del concepto de reflexión en la formación de profesores. Su discusión se configura en una interesante mixtura de afirmaciones fundadas con opiniones personales acerca de diversos niveles de reflexión, los que incluyen los niveles de reflexión técnica, descriptiva, dialógica y crítica (p.45). Relacionan la reflexión técnica con la racionalidad técnica tan criticada por Schön (1983), mientras que la reflexión descriptiva, la dialógica y la crítica son asociadas con la reflexión en la acción de Schon. Para ellos, la reflexión en la acción es interpretada como "la contextualización de múltiples puntos de vista", considerando los cuatro niveles de reflexión (Hatton \& Smith, p. 45). Su debate sobre el lugar de la reflexión en la formación de profesores es conducido y apoyado por un proyecto de pequeña escala llevado a cabo en la Universidad de Sidney. Una de sus afirmaciones apunta a la consideración de que cuando se incorpora la reflexión a un programa de formación inicial de profesores, lo que se requiere es un cambio paradigmático:

Una aproximación críticamente reflexiva demanda una ideología de formación de profesores distinta a las tradicionalmente empleadas, las que usualmente involucran modelos de "mejores prácticas", énfasis en competencias y conflictos no reconocidos entre los ideales institucionales y socialización en los lugares de 
trabajo." Tienen que haber cambios en el énfasis y la creación de oportunidades que establezcan condiciones de apoyo apropiadas para promover en los estudiantes distintos tipos de reflexión (p. 38).

La utilización de términos tales como "conflictos no reconocidos" y "cambios en los énfasis" podría ser un intento engañoso para minimizar la importancia de adoptar un enfoque de reflexión a lo largo de todo un programa de formación docente, engañoso en el sentido que - como sugiere Schön (1995) lo que se requiere es de un cambio profundo en la epistemología.

Una significativa contribución en este artículo aparece en la búsqueda que hacen los autores por hallar evidencias de práctica reflexiva, y ofrecen listas de características de escritura descriptiva, de reflexión descriptiva, de reflexión dialógica y de reflexión crítica (pp. 40-41, 48-49). Su (foco) (enfoque) en la búsqueda de evidencias de reflexión parece estar en los escritos de algunos estudiantes sobre "el proceso de la planificación, desarrollo, implementación y evaluación de clases" (p.42). Ellos reconocen que esto se trata de reflexión sobre la acción, pero esperan que la reflexión en la acción también haya estado presente: "es probable, sin embargo, que la "reflexión en la acción" haya ocurrido mientras los eventos se desarrollaban originalmente, en que los estudiantes estuvieran pensando en las razones para que ocurriera lo que estaban experimentando mientras ocurría" (p. 42). Ofrecen entonces un comentario adicional acerca de la tensión entre reflexión y los supuestos presentes: "el género académico tradicional se caracteriza por tener características que, en muchos casos, son la antítesis del estilo de escritura personal, exploratorio, tentativo y en ocasiones indeciso, que podría ser identificado como reflexivo" (p. 42).

Mi interpretación del argumento presentado por Hatton y Smith reconoce que ellos logran ver que la reflexión en la acción es un fenómeno complejo que surge en el contexto de la acción profesional. Su conclusión indica que están empeñados a ver la reflexión como un proceso que se desarrolla a través de una jerarquía de niveles que va desde el técnico, pasando por el descriptivo hasta un nivel crítico. Sólo entonces es que el profesional que está adquiriendo experiencias está listo para reflexionar en la acción. Su conclusión se nutre de un estudio sobre profesores en formación, pero no presentan una evidencia que respalde la jerarquía de los niveles de reflexión. Ellos enfatizan que sus estudiantes necesitan desarrollar habilidades metacognitivas para poder avanzar hacia una reflexión en la acción, mas parecen reacios a admitir que los profesores en formación podrían experimentar momentos de replanteamientos mientras enseñan a estudiantes reales en salas de clases reales.

\section{Carol Rodgers: volviendo a Dewey e ignorando a Schön.}

Rodgers (2002) hace una valiosa contribución a nuestra comprensión del concepto de reflexión al volver al trabajo de Dewey. Su propósito merece atención: "Durante los últimos 15 años, la reflexión ha sufrido una pérdida de significado. Al volverse un todo para todos, ha perdido la capacidad de ser vista" (p. 843). Este extenso artículo parece ser una excelente introducción al trabajo de Dewey sobre el pensamiento 
reflexivo. De forma interesante, a pesar del rol que tuvo Schön (1983) en volver nuestra atención a Dewey y al concepto de reflexión, ella ignora la oportunidad de interpretar el trabajo de Schön a la luz del trabajo de Dewey.

Rodgers describe y discute cuatro criterios de reflexión, extraídos de Dewey: (1) un proceso de toma de significado, (2) una forma rigurosa de pensamiento, (3) un proceso que ocurre en comunidad y, (4) un grupo de cuatro actitudes: entrega de todo corazón, ser directo, apertura de mente y responsabilidad. "Las cuatro actitudes comprenden los aspectos constituyentes esenciales de lo que Dewey llama preparación para participar en la reflexión" (p. 862). Rodgers resume luego su argumento afirmando que ha demostrado que

la reflexión no es un fin en sí mismo, sino una herramienta o vehículo utilizado en la transformación de experiencias puras en una teoría llena de significado, basada en la experiencia, informada por las teorías existentes y que sirve al propósito más amplio del crecimiento moral del individuo y de la sociedad. (p. 863)

La posición de Rodgers en torno a que la reflexión es un medio y no un fin en sí mismo es una señal importante para los formadores de profesores. Su artículo incluye descripciones de experiencias de profesores, pero no se refiere de forma explícita a las prácticas profesionales de aquellos que están aprendiendo a enseñar. Sin embargo, resulta altamente importante y apropiado ver el papel de las prácticas profesionales desde la perspectiva de la transformación de la experiencia en "teoría que se fundamenta en la experiencia" e "informada por la teoría existente" (p. 863).

\section{Barbara Larrivee: Convirtiéndose en un profesor críticamente reflexivo.}

Las consideraciones de Larrivee (2000) en torno a cómo una persona se desarrolla como un profesional críticamente reflexivo añade poco a las ideas ya exploradas en el trabajo de Schön, al que - tal como Rodergs- no hace referencia. Sin embargo, resulta importante abordar aquí este artículo, porque ilustra el grado al que la idea de "volverse un profesor críticamente reflexivo" se ha vuelto un movimiento en sí mismo, bastante independiente del trabajo de Schön, con sujetos que construyen interpretaciones disímiles con respecto a la reflexión. Larrivee (2000) señala lo siguiente:

La reflexión crítica es el atributo distintivo de los profesionales reflexivos. El término reflexión crítica, tal como se desarrolla aquí, amalgama la indagación crítica, la consideración consciente de las implicaciones éticas y las consecuencias de la práctica de la enseñanza con la reflexión sobre sí mismo, la examinación profunda de las creencias personales, y los supuestos sobre el potencial humano y el aprendizaje. (p.293)

Llama la atención que Larrivee considera el trabajo de Dewey (1933) sobre el pensamiento reflexivo y el trabajo de Argyris (1990) sobre las creencias auto-generadas, mas 
no probadas, en su esfuerzo por elevar la reflexión crítica al estatus de característica definitoria de un docente reflexivo: "Los profesionales reflexivos desafían los supuestos y cuestionan las prácticas existentes, por lo que continuamente cuentan con nuevos lentes(...) para ver su práctica y modificar sus perspectivas" (p.296). Para ella, habrían tres prácticas esenciales para convertirse en un profesional reflexivo, las que son: "apartar tiempo para reflexionar solitariamente", "convertirse en un "solucionador de problemas" perpetuo" y "cuestionar el statu(s) quo" (pp.296-297). Estas prácticas son presentadas como procesos para los que uno debe tomar tiempo, y por tanto, difieren dramáticamente de la discusión que expone Schön sobre la reflexión en la acción como proceso que involucra replanteamientos espontáneos e intuitivos, en lugar de controlados y deliberados. Más adelante en su argumento, Larrivee parece acercarse más al trabajo de Schön al hablar de planteamiento) y replanteamiento. Sin embargo, acto seguido, sugiere que "al desafiarse a sí mismos a crear nuevos puntos de ventaja, los profesores pueden asignar nuevos significados a las situaciones de aula que confrontan" (p.299). Aquí vemos nuevas maneras de ver situaciones profesionales presentadas como si surgieran de forma aislada y después de la acción, en lugar de verlas como algo que surge desde una "conversación reflexiva con la situación" (Schön 1983:167).

Las muchas y significativas diferencias que se dan entre las posturas de Larrivee y Schön con respecto a la reflexión se vuelven más notorias cuando se comparan las siguientes citas. Schön da cuenta de la indagación en el contexto de reflexión en la acción en los siguientes términos:

$\mathrm{Al}$ mismo tiempo en que el investigador trata de formar la situación al interior de su marco referencial, debe permanecer abierto a las respuestas de la situación. Debe estar dispuesto a adentrarse en nuevas confusiones e incertidumbres. Por lo tanto, tiene que ser capaz de adoptar una doble visión. Debe actuar de acuerdo a la visión que ha adoptado, pero sabiendo que siempre puede revisarla, de hecho, debe revisarla más tarde para poder dar sentido a su conversación con la situación... Mientras el riesgo de la incertidumbre se incrementa, también aumenta la tentación de tratar esa visión como si fuera la realidad. Así y todo, si el investigador mantiene su doble visión, incluso al profundizar su compromiso con el marco seleccionado, aumenta sus oportunidades de llegar a una coherencia mayor y más amplia entre artefacto e idea. (p. 164)

La referencia de Schön a una "visión doble" — mantener simultáneamente marcos de referencia antiguos y nuevos mientras se busca la evidencia de que uno es más productivo que el otro-enfatiza su enfoque en la importancia de la acción profesional que se lleva a cabo de manera metacognitiva, consciente de los supuestos que están operando cuando se decide a actuar de una manera particular.

En contraste, Larrivee utiliza un lenguaje bastante dramático para describir su visión del proceso de reflexión crítica como algo cercano a lo heroico:

Si por el contrario estamos en condiciones de enfrentar el conflicto, dejando de lado lo que nos resulta familiar, nos permitimos experimentar la incertidumbre. 
Este no saber nos arroja a un caos. En esta fase, si nos movemos "hacia el ojo de la tormenta", logramos pasar la confusión y una nueva y más profunda comprensión emerge, encaminándonos hacia la fase de reconciliación. En esta última etapa, habremos experimentado un cambio en nuestras maneras de pensar y de sentir. Vemos...las cosas desde una perspectiva diferente. Nos involucramos en nuevos patrones de pensamiento y accedemos a nuevas herramientas y estrategias para responder más apropiadamente a las situaciones de aula. (p.305)

Aunque esta descripción tiene algunas semejanzas con el trabajo que hace varios años Schön había hecho sobre reflexión en la acción (varios años antes), Larrivee no hace referencia alguna al trabajo de Schön, como tampoco ofrece evidencia para respaldar sus interpretaciones. La perspectiva de Schön está respaldada con una variedad de estudios de caso, y él enfatiza la importancia de poner a prueba las nuevas formas de pensar, sin embargo Larrivee parece asumir que una nueva perspectiva siempre supondrá una mejora.

\section{Lograr replanteamientos mientras se enseña y se aprende a enseñar: El potencial de escuchar en momentos de incertidumbre y sorpresa}

Luego de haber revisado una pequeña porción de la extensa literatura sobre reflexión en el contexto de la formación de profesores, se hace necesario sacar conclusiones y determinar claramente qué es lo que se está diciendo. No pretendo discutir la reflexión en las muchas y diferentes maneras en que el termino puede ser interpretado. Hay tres puntos que en realidad son de particular interés para mí:

1. El libro de 1983 de Donald Schön es generalmente reconocido como la fuente principal de las referencias a la importancia de la reflexión, de la reflexión crítica y cómo llegar a ser un profesional reflexivo, en el ámbito de la formación inicial docente. Schön no ocupó el término crítico, el que parece haber sido añadido después, cuando la palabra reflexion por si sola no producía los efectos esperados. La adición de este término y la posterior construcción de niveles de reflexión constituyen un ejercicio interesante, aunque hay poca evidencia de que haya cambiado la manera en que las personas aprenden a enseñar.

2. Las ideas de Schön han sido extensamente discutidas y criticadas (Eraut, 1995, es un significativo ejemplo) pero se ha visto poco esfuerzo en identificar de qué manera el trabajo de Schön podría contribuir a la educación de nuevos profesionales, incluyendo profesores.

3. Mi propia interpretación es que Schön nos estaba invitando a considerar más cuidadosamente y en mayor detalle la manera en que tanto los nuevos profesionales 
como aquéllos más experimentados aprenden de sus propias experiencias de acción profesional. Aunque hay una amplia variedad de referencias a la reflexión en la literatura de formación docente, encontramos muy pocos ejemplos de discusiones que consideren las experiencias de práctica profesional en que los profesores principiantes logran aprender de su experiencia personal, junto a la guía de un mentor. El segundo libro de Schön (1987) se enfoca en el aprendizaje desde la experiencia en interacción con un mentor.

Jamás pretendería decir que Schön tiene la última palabra en lo que se refiere a cómo los profesionales aprenden de su experiencia. Sin embargo, se ha vuelto una preocupación importante para mí (el) ver el grado al que ha llegado la poca o incluso inexistente conexión entre las clases en la universidad y las experiencias de práctica profesional en escuelas, en el contexto de la formación inicial de profesores.

He trabajado por 35 años en el mismo programa de postgraduado, de ocho meses de duración, de formación inicial docente. Se podría decir que a excepción de dos años, la estructura del programa podría ser descrita como tradicional. Los dos años revolucionarios resultaron ser demasiado revolucionarios para mis colegas de esa época. Mientras oigo las experiencias de práctica de mis estudiantes, escucho una conclusión universal: las prácticas en la escuela son el elemento más crucial de todo el programa de formación docente. Con mayor frecuencia, mis estudiantes sugieren que tanto los contenidos de los cursos como los trabajos escritos deben tener una conexión más directa con las experiencias de práctica en las escuelas.

¿Qué podríamos lograr al establecer conexiones más directas entre estos dos contextos de aprendizaje tan distintos - los cursos universitarios y la práctica - en los programas de formación de profesores? Mientras (que) los profesores más experimentados tienen acceso a diversos recursos de desarrollo profesional (como conferencias, talleres y comunidades de aprendizaje al interior de los colegios), siempre serán sus propias clases y las interacciones diarias con los estudiantes las que representan las mejores oportunidades de aprendizaje y cambio que mejora(n) el ejercicio profesional. Posiblemente la conocida división entre teoría y práctica ha resultado ser demasiado potente y penetrante, pues las clases que vemos en la escuela muy rara vez logran dar cuenta explícita y genuinamente de un aprendizaje desde la experiencia. Es mucho más común aprender por medio de la lectura o por medio de poner mayor atención a aquellos profesores de los que se espera sepan mucho más que sus estudiantes.

A pesar de las muchas elaboraciones a partir del trabajo de Schön en el ámbito de la reflexión en la acción, sostengo que vemos poca evidencia de sus ideas en las discusiones sobre reflexión en la formación docente debido a que se nos hace dificil construir enlaces directos entre los cursos de educación y las experiencias de práctica. Las clases de la universidad usualmente parecen estar colocadas en una posición a lo que Schön llamaba los "Alturas" (1983, p.42), mientras que los lugares de práctica requieren que los candidatos a profesor, actúen en los "terrenos bajos y cenagosos donde las situaciones son "líos" confusos, incapaces de una solución técnica." Aquellos que enseñan cursos básicos de educación en las universidades rara vez son los mismos que observan y acompañan a sus estudiantes en sus prácticas en la escuela, por lo que 
tienden a olvidar lo complicado e irrealista que resulta hablar de "aplicar la teoría en la práctica.”

En mi propio trabajo con la perspectiva de Schön, he podido darme cuenta que debemos ayudar a los profesores en formación a aprender a cómo aprender (mejor) de sus experiencias en la sala de clases. Hacer esto no es fácil (Russell, 2005) puesto que casi no puede hacerse por medio de exposiciones o relatos. Enseñar a aprender de la experiencia requiere de un modelado explícito y deliberado por parte de los formadores de docentes. Una de las contribuciones de Schön es la de comprender la importancia de nombrar los problemas cuidadosamente antes de intentar resolverlos. Otra de sus contribuciones consiste en la importancia de una conversación reflexiva con las situaciones en la sala de clases. En este sentido, los profesionales necesitan poner especial atención a los momentos de incertidumbre, sorpresa y perplejidad, puesto que pueden estimular replanteamientos y generar nuevas maneras, posiblemente más productivas de interpretar los comportamientos de los estudiantes, y generar perspectivas que podrían llevar a cambios en el curso de acción.

\section{La complejidad de un cambio paradigmático: un ejemplo personal}

En la introducción a este capitulo comenté que estuve trabajando durante 3 años con profesores con experiencia, antes de empezar a enseñar en un programa de pedagogía. Durante el último de esos tres años, trabajamos junto a otro colega con un grupo de cinco profesores de historia y el director del colegio para mostrarles cómo identificar patrones en las transcripciones de clases grabadas, y luego trabajarlos para construir patrones alternativos de interacciones profesor-estudiante que pudiesen resultar más productivas (Ireland \& Russell, 1978). Los profesores llegaron a dos conclusiones generales: 1) no se habían dado cuenta de cuánto hablaban en cada clase que daban, y 2) no imaginaban lo difícil que sería alejarse de ese patrón de interacción dominante, en la que los profesores son casi los únicos que hablan. La disposición de estos profesores a abrir su modo de enseñanza a los ojos de otros, y las conclusiones a las que llegaron me parecieron dignas de respeto y admiración.

Cuatro meses más tarde, me encontré frente a mi primer curso de educación de profesores, con la fuerte determinación de hacer lo posible para reducir la cantidad de tiempo que hablaría. Como aquellos profesores de historia, no tenía idea de cómo poder remplazar la charla con alternativas más productivas. En retrospectiva, en ese primer año de trabajo en la formación de profesores pude experimentar muchos momentos de lo que más tarde Schön llamaría reflexión en la acción. Me esforcé por desarrollar nuevos patrones de interacción, y los más productivos a los que llegué fueron aquellos de escuchar a quienes estaba enseñando. Me comencé a reunir con pequeños grupos de estudiantes para llegar a conocerlos más personalmente, e instauré una evaluación de mi enseñanza a la mitad del curso, en la que los estudiantes respondían libremente bajo las categorías de fortalezas, debilidades y conclusiones. Al incorporar los resultados a mis clases, se abrieron espacios de discusión, y en uno de ellos me 
encontré explicando por qué había decidido reducir la cantidad del tiempo que les hablaba en clases. La respuesta de uno de mis estudiantes resultó inolvidable, cuando exclamó exasperado:” ¿Por qué no nos dijo que no iba a decírnoslo?”

Al cumplir 35 años de trabajo en el campo de la formación inicial docente, estoy profundamente consciente de la estabilidad de las prácticas de enseñanza en general, y en la formación pedagógica en particular. He tenido la fortuna de investigar y escribir en el contexto mismo de la formación de profesores. Con el tiempo, he encontrado que mi modo de enseñanza con los profesores en formación ha cambiado dramáticamente en la medida que he escuchado a los que enseño, que he estudiado mis propias prácticas de enseñanza (Schuck \& Russell, 2005) y que he ido encontrando ideas de enseñanza innovadoras que he ido adaptando a la formación de profesores y haciéndolas parte de mi ejercicio profesional.

El programa en el que he trabajado ha visto muchos cambios durante las últimas 3 décadas, y sin embargo en muchos aspectos no ha cambiado nada. Los periodos de práctica profesional ahora son un poco más largos. Luego de la incorporación de la palabra reflexión, los trabajos y clases han cambiado en el vocabulario que utilizan, pero no hay suficiente evidencia que haya habido cambios profundos en los resultados que se obtienen. En (el año) 1997, una restructuración radical del programa permitió enviar a los estudiantes a prácticas extendidas en los colegios (14 semanas) adelantándose a los cursos regulares de educación. Nueve meses más tarde, un grupo de académicos en la universidad votó para abandonar ese cambio radical. Esa experiencia permitió generar una versión preliminar de la Figura 1 (ver anexo), la que ilustra algunos de los supuestos al interior de dos paradigmas muy distintos de cómo entender la manera en que aprenden los profesores.

Los cambios de paradigma en la enseñanza toman tiempo, pero fundamentalmente, requieren estar dispuesto a enfrentar riegos y estar abierto a cometer errores. La reflexión en la acción, impulsada por una "conversación reflexiva con la situación” (Schön, 1983, p. 163) puede inspirar maneras más productivas de interpretar la complejidad de lo que ocurre en nuestras salas de clase, sin embargo, no necesariamente todo acto de replanteamiento genera cambios productivos. Del mismo modo, cuando un replanteamiento nos inspira a asumir el riesgo de cambiar una práctica, muy rara vez esa nueva acción se desarrollará a la perfección la primera vez que se haga. Requerirá de paciencia en su afinamiento y de más conversaciones con la situación antes de que llegue a formarse como un hábito más productivo que el anterior. En una de sus últimas publicaciones, Schön comentó de la necesidad de un nuevo tipo de conocimiento académico (scholarship):

La epistemología apropiada para el nuevo saber académico debe hacer espacio para la reflexión en y sobre la acción del profesional. Debe dar cuenta y legitimar no sólo el uso del conocimiento producido en la universidad, sino que la generación de saberes practicables construidos por el profesional, en la forma de modelos o prototipos que luego puedan ser trasladados - por medio de una transferencia reflexiva- a nuevas situaciones prácticas. Este nuevo saber académico requiere de una epistemología de la práctica reflexiva, la que incluye 
lo que Kurt Lewin describió como investigación-acción. Sin embargo, tanto en la universidad investigativa moderna como en otras instituciones de educación superior influenciadas por ella, la práctica reflexiva en general y la investigación acción en particular, están destinadas a enfrascarse en una batalla contra la epistemología dominante de la racionalidad técnica. (Schön, 1995, p. 34)

Schön no se muestra muy optimista con respecto a las posibilidades para el cambio paradigmático en universidades y escuelas. Los objetivos a los que he apuntado en este capitulo se relacionan con un recordatorio a los lectores de que una perspectiva de práctica reflexiva no es algo simple de alcanzar, ya sea a nivel personal o institucional. No deberíamos esperar que los estudiantes adopten nuevos marcos de referencia y cambien hábitos antiguos si no lo hemos experimentado en nosotros mismos primero, de tal modo que comprendamos profundamente en los hechos en lugar de palabras aquello que les estamos pidiendo a los estudiantes que hagan.

Junto a varios otros colegas Canadienses desarrollamos la Figura 1 (ver anexo) con el propósito de contrastar el ya familiar o tradicional enfoque a la formación docente de "teoría transferida a la práctica", con una que incorpora el aprendizaje desde la experiencia junto al aprendizaje en las aulas universitarias (Martin, Russell, Bullock, O'Connor \& Dillon, 2012). La columna "práctica y teoría" ilustra la magnitud del cambio que debe haber en los supuestos y las prácticas para modificar un paradigma que se aleje de la racionalidad técnica o de la "teoría por sobre la práctica."

\section{Acciones personales basadas en una reflexión en la acción}

Para continuar el análisis de la reflexión en la acción de Schön, y la desatención a sus contribuciones específicas que se ve en la literatura sobre formación docente, quiero presentar una serie ejemplos de reflexión en la acción que han resultado en contribuciones significativas a mi propia práctica como formador de profesores. Debiera recordar a los lectores que antes de publicar El profesional Reflexivo, Schön publicó varios libros junto a Argyris (Argyris \& Schön, 1974), que se enfocaron en los desafíos organizacionales e interpersonales asociados al hecho de que la mayoría de las personas demuestran una escisión significativa entre sus teorías adoptadas (lo que dicen que hacen) y sus teorías en uso (teorías que pueden ser inferidas al observar sus acciones). Este es un punto de particular importancia para la formación inicial y capacitación en servicio de profesores, donde lo que hacemos y lo que decimos son siempre notados y comparados por nuestros estudiantes. Aquellos estudiantes que aprenden a enseñar rápidamente se sienten frustrados cuando se les dice que no tienen que darles charlas a sus estudiantes; que alguien te diga que no debes hacer lo que esa misma persona está haciendo es una contradicción obvia y frustrante.

No creo estar bien calificado para responder la amplia pregunta, ¿Qué es el aprendizaje?, sin embargo hago aquí la pregunta en el contexto de individuos que están aprendiendo a ser profesores. Cada persona que se forma como docente empieza ese 
proceso con una poderosa carga de aprendizajes por observación. Cada uno de ellos conoce (por observación, no por formación deliberada) los hábitos que muestran los profesores, y lleva a cabo una imitación de esos hábitos en su sala de clases, aunque de forma un tanto incómoda al principio. Aún así, la mayoría de los profesores principiantes asume que sabe poco acerca de la enseñanza. Aunque hayan adquirido muchos hábitos de enseñanza a lo largo de su experiencia como estudiantes, en realidad saben muy poco acerca de las lógicas y racionalidades de enseñanza - las perspectivas acerca del aprendizaje que tenían los profesores de quienes adquirieron ciertos hábitos, de manera indirecta e involuntariamente. Me gusta utilizar el término "marco" para referirme a estas perspectivas puesto que se relaciona bien con la idea de "replanteamiento" de Schön. Cabe notar que la discusión de Schön sugiere que es cuando los eventos son desconcertantes, sorprendentes o inciertos que podemos re-enmarcar o replantear el problema, al ser inspirados a ver las cosas desde nuevas perspectivas por medio de una conversación reflexiva con los materiales de la situación. Aprender de la experiencia es un proceso complejo que resulta bastante diferente al aprendizaje desde un libro o de las experiencias de otro. Cuando un profesor aprende de su experiencia, ese aprendizaje tiene que ser visto simultáneamente desde dos perspectivas: hábitos y marcos. Si aprendemos de la experiencia, actuamos de manera distinta la próxima vez que tenemos una experiencia similar y pensamos distintos acerca de cómo, por qué y para qué estamos actuando de esa manera particular en ese contexto de acción.

Como todos los padres saben en algún sentido, el aprendizaje que tiene un hijo a través de la experiencia también tiende a ser mucho más potente que lo que se aprende por medio de un libro o por las indicaciones que alguien diga. Aunque no fui el primero en usar este ejemplo, siempre recurro a la idea de "la estufa está caliente" para ilustrar tres tipos de aprendizaje. Cuando nos dicen que la estufa está caliente, posiblemente la estufa se vea exactamente igual a cuando esta fría; aparentemente no hay ninguna razón para actuar de modo distinto alguno. Cuando vemos que alguien más toca una estufa caliente y salta hacia atrás rápidamente, la estufa puede lucir exactamente igual, pero la reacción de la otra persona puede representar un mensaje de alerta que resulta más fácil de internalizar. Cuando nosotros mismos tocamos una estufa caliente y saltamos hacia atrás, nos damos cuenta que aunque la estufa no se vea distinta, en realidad sí estaba muy diferente, entonces aprendemos de primera mano la lección de que deberíamos ser cuidadosos al acercarnos a una estufa.

En los 35 años que llevo aprendiendo de la experiencia como educador de profesores, el cambio ha venido lentamente en ciertos momentos, y en otros han ocurrido cambios más rápidamente. El libro de 1983 de Schön me proveyó una de las primeras instancias importantes para el replanteamiento de mis experiencias durante los 6 años anteriores como formador de profesores. Estaba perplejo ante el hecho de que cuando visitaba las prácticas de clase de mis estudiantes, veía muy pocas evidencias de comportamientos que pudieran haber sido inspirados en sus cursos de pedagogía. Estaban enseñando del mismo modo en que les habían enseñado a ellos. La reflexión en la acción y el concepto de replanteamiento se convirtieron en una poderosa manera de avance para mí. De forma gradual mis marcos interpretativos cambiaron, también lo 
hicieron mis hábitos, mientras encontraba nuevos recursos y me arriesgaba a probar nuevos patrones de interacción en mis clases de formación pedagógica.

\section{Los peligros, trampas y la promesa no cumplida del profesional reflexivo}

El libro de Schön de 1983 dio la pauta para que la profesión de la formación de profesores adoptara la palabra reflexión e intentara formar profesores como profesionales reflexivos. En el genuino sentido de la racionalidad técnica, la teoría pudo haber sonado llamativa, pero su traducción a la práctica no ha logrado el cambio paradigmático que ésta implica. En el caso de la teoría acerca de la reflexión, como Schön la presentó, ha sido simultáneamente ignorada y también reinterpretada en una variedad de formas para adaptarse a estructuras y propósitos ya existentes. De este modo, lo que veo en las innumerables formas en que la educación de profesores ha respondido al camino de la reflexión planteado por Schön es una gran promesa no cumplida. Hay una serie de peligros y trampas que surgen cuando los profesionales cambian su vocabulario, pero no sus acciones. Los programas de formación inicial docente son pocas veces recordados de manera muy favorable por aquellos que están aprendiendo a enseñar. (Posiblemente)Probablemente esto ocurre porque los primeros años de enseñanza son tan abrumadores y requieren de tanto aprendizaje personal de la experiencia (Schuck, Aubusson, Buchanan \& Russell, 2012), que los profesores ya más experimentados concluyen que sus programas de pedagogía no sirvieron para prepararlos adecuadamente para el trabajo real de los profesores.

Tal como ocurre con las prácticas de enseñanza en general (investigado en profundidad por Sarason, 1971), las prácticas que se dan en la formación de profesores tienden a ser muy estables. Sólo un cambio paradigmático de los supuestos fundamentales y de las prácticas de formación docente lo que también va a acompañado de nuevos riesgos y peligros podría llegar a tender vínculos significativos entre lo que se presenta como teoría en las clases de educación y lo que se experimenta en las prácticas en los colegios. Schön sugirió nuevas perspectivas y rumbos con respecto a desafíos permanentes en la formación de profesores. Aún nos queda pendiente alcanzar la promesa de la perspectiva del profesional reflexivo. 


\section{Referencias}

Argyris, C., \& Schön, D. A. (1974). Theory in practice: Increasing professional effectiveness. San Francisco, CA: JosseyBass.

Calderhead, J. (1992). The role of reflection in learning to teach. En L. Valli (Ed.), Reflective teacher education: Cases and critiques (pp. 139-146). Albany, NY: State University of New York Press.

Eraut, M. (1995). Schön shock: a case for refraining reflection-in-action? Teachers and teaching: Theory and practice, 1(1), 9-22.

Fendler, L, (2005). Teacher reflection in a hall of mirrors: Historical influences and political reverberations. Educational Researcher, 32(3), 16-25.

Hatton, N., \& Smith, D. (1995). Reflection in teacher education: Towards definition and implementation. Teaching and Teacher Education, 11, 33-49.

Ireland, D., \& Russell, T. (1978). The Ottawa Valley teaching project. Journal of Curriculum Studies, 10(3), 206-208.

Larrivee, B. (2000). Transforming teaching practice: Becoming the critically reflective teacher. Reflective Practice, 1, 293-307.

Lortie, D. A. (1975). Schoolteacher: A sociological study. Chicago, IL: University of Chicago Press.

Martin, A. K., Russell, T., Bullock, S., O’Connor, K., \& Dillon, D. (2012, May). Alternative conceptual frameworks for teacher education programs in Canada. Documento presentado en la reunión de la Canadian Association for Teacher Education, Wilfred Laurier University, Waterloo, ON.

Rodgers, C. (2002). Defining reflection: Another look at John Dewey and reflective thinking. Teachers College Record, 104, 842-866.

Russell, T. (2005a). Can reflective practice be taught? Reflective Practice, 6, 199-204.

Russell, T. (2005b). The place of the practicum in preservice teacher education programs: Strengths and weaknesses in alternative assumptions about the experiences of learning to teach. In G. Hoban (Ed.), The missing links in teacher education design (pp. 135-152). Dordrecht, The Netherlands: Springer.

Sarason, S. B. (1971). The culture of the school and the problem of change. Boston, MA: Allyn and Bacon.

Schön, D. A. (1983). The reflective practitioner: How professionals think in action. New York, NY: Basic Books.

Schön, D. A. (1987). Educating the reflective practitioner: Toward a new design for teaching and learning in the professions. San Francisco, CA: Jossey-Bass.

Schön, D. A. (Ed.) (1991). The reflective turn: Case studies in and on educational practice. New York, NY: Teachers College Press.

Schön, D. A. (1995). The new scholarship requires a new epistemology. Change, 27(6), 26-34.

Schuck, S., \& Russell, T. (2005). Self-study, critical friendship, and the complexities of teacher education. Studying Teacher Education, 1, 107-121.

Schuck, S., Aubusson, P., Buchanan, J., \& Russell, T. (2012). Beginning teaching: Stories from the classroom. Dordrecht, The Netherlands: Springer.

Valli, L. (Ed.). (1992). Reflective teacher education: Cases and critiques. Albany, NY: State University of New York Press.

Zeichner, K. (1996). Teachers as reflective practitioners and the democratization of schools reform. In K. Zeichner, S. Melnick, \& M. L. Gomez (Eds.), Currents of reform in preservice teacher education (pp. 199-214). New York: Teachers College Press. 
Figura 1. Comparación de perspectivas sobre el diseño de formación docente.

\begin{tabular}{|c|c|c|}
\hline & $\begin{array}{l}\text { Perspectiva de teoría aplicada a } \\
\text { la práctica }\end{array}$ & Perspectiva de práctica y teoría \\
\hline $\begin{array}{l}\text { ¿Cuál es la naturaleza } \\
\text { del saber docente? }\end{array}$ & $\begin{array}{l}\text { El conocimiento que los } \\
\text { candidatos necesitan enseñar es } \\
\text { proposicional, y su significado } \\
\text { puede ser comprendido sin } \\
\text { experiencia en el aula. }\end{array}$ & $\begin{array}{l}\text { El saber práctico de un profesor } \\
\text { incluye imágenes, sentimientos, } \\
\text { valores y experiencias, así como } \\
\text { conocimientos expresados en } \\
\text { proposiciones. La teoría no puede } \\
\text { ser comprendida a cabalidad sin } \\
\text { experiencias practicas personales. }\end{array}$ \\
\hline $\begin{array}{l}\text { ¿Qué es lo que } \\
\text { los candidatos } \\
\text { saben acerca de la } \\
\text { enseñanza? }\end{array}$ & $\begin{array}{l}\text { Los estudiantes saben muy } \\
\text { poco acerca de la enseñanza. } \\
\text { Vienen a aprender teoría y } \\
\text { otras generalidades acerca de } \\
\text { la enseñanza, las que después } \\
\text { tendrán que expresar durante sus } \\
\text { periodos de práctica profesional. }\end{array}$ & $\begin{array}{l}\text { Los estudiantes tienen una } \\
\text { extensa acumulación de imágenes } \\
\text { acerca de la enseñanza que guían } \\
\text { sus primeras acciones. Les falta } \\
\text { acceso al tipo de pensamiento } \\
\text { que acompaña las acciones } \\
\text { de enseñanza. Sólo durante } \\
\text { experiencias prácticas pueden } \\
\text { aprender a enlazar pensamiento } \\
\text { y acción. }\end{array}$ \\
\hline $\begin{array}{l}\text { ¿Cuán fuertes son los } \\
\text { valores educacionales } \\
\text { de los candidatos a } \\
\text { docente? }\end{array}$ & $\begin{array}{l}\text { Los valores de los estudiantes } \\
\text { para mejorar la enseñanza son } \\
\text { frágiles, débiles y fácilmente } \\
\text { erosionados por la exposición a } \\
\text { malas prácticas en los colegios. } \\
\text { Las clases de pedagogía deberían } \\
\text { ayudarles a resistir la inclinación a } \\
\text { adoptar prácticas acríticamente. }\end{array}$ & $\begin{array}{l}\text { Los valores pedagógicos de } \\
\text { los estudiantes son fuertes y } \\
\text { están basados en las imágenes } \\
\text { que tienen de sus profesores } \\
\text { anteriores. Aplican estos valores } \\
\text { a las prácticas existentes de las } \\
\text { escuelas, del mismo modo en } \\
\text { que los aplican a su análisis de } \\
\text { cómo se enseñaron sus cursos de } \\
\text { educación. }\end{array}$ \\
\hline $\begin{array}{l}\text { ¿Cuánta experiencia } \\
\text { requiere un profesor } \\
\text { principiante? }\end{array}$ & $\begin{array}{l}\text { Periodos cortos de experiencia } \\
\text { ( } 2 \text { a } 3 \text { semanas) son un mínimo } \\
\text { suficiente para practicar los } \\
\text { conocimientos adquiridos en las } \\
\text { clases sobre educación. }\end{array}$ & $\begin{array}{l}\text { La práctica extendida apoya y } \\
\text { consolida los aprendizajes en } \\
\text { experiencia y prepara de mejor } \\
\text { manera a los estudiantes para } \\
\text { su primer año como profesores, } \\
\text { desarrollando la confianza en si } \\
\text { mismos. }\end{array}$ \\
\hline $\begin{array}{l}\text { ¿Pueden aprender } \\
\text { de sus propias } \\
\text { experiencias? }\end{array}$ & $\begin{array}{l}\text { A los estudiantes se les debe } \\
\text { decir cómo pensar acerca de la } \\
\text { teoría y la experiencia. Aquellos } \\
\text { con mayor experiencia tienen que } \\
\text { contarles de qué modo se puede } \\
\text { mejorar la escuela. }\end{array}$ & $\begin{array}{l}\text { Los estudiantes pueden y } \\
\text { deben pensar por si mismos. } \\
\text { También valoran el aprender de } \\
\text { las experiencias de otros. Sus } \\
\text { referentes significativos incluyen a } \\
\text { académicos, profesores ayudantes } \\
\text { y otros compañeros de curso. }\end{array}$ \\
\hline $\begin{array}{l}\text { ¿Cómo puede } \\
\text { mejorar un profesor } \\
\text { principiante conforme } \\
\text { gana experiencia? }\end{array}$ & $\begin{array}{l}\text { Los candidatos mejoran con } \\
\text { la experiencia, a medida que } \\
\text { sus mentores les proveen } \\
\text { tips prácticos que pueden ir } \\
\text { acumulando. (Algunos profesores } \\
\text { llegan a asumir que lo que } \\
\text { se enseña en los cursos de } \\
\text { educación suena bien pero es } \\
\text { irrealista). }\end{array}$ & $\begin{array}{l}\text { Conforme los profesores guía } \\
\text { proveen una crítica constructiva, } \\
\text { los candidatos aprenden a } \\
\text { criticar sus propias prácticas. Las } \\
\text { mejoras también son guiadas } \\
\text { por sus valores personales y sus } \\
\text { razones para entrar a la carrera de } \\
\text { pedagogía. }\end{array}$ \\
\hline
\end{tabular}




\section{Notas}

1. Nota del Traductor: los años citados corresponden a la fecha de publicación de los originales en Inglés. 